\title{
Cyanosis As a Signal of Perinatal Stroke: Findings at Ultrasound and MRI
}

\author{
Yu Chuan Chang ${ }^{1}$; Chun Yi Lee ${ }^{1}$; Chao Huei Chen ${ }^{2}$; Yi Chia Ku ${ }^{2}$; jiaan Der Wang ${ }^{2,3,}$ \\ ${ }_{1}^{1}$ Department of Pediatrics, Chang Bing Show Chwan Memorial Hospital, Changhua, Taiwan, Republic of China \\ ${ }_{3}^{2}$ Department of Pediatrics, Taichung Veterans General Hospital, Taichung, Taiwan, Republic of China \\ ${ }^{3}$ School of Medicine, China Medical University, Taichung, Taiwan, Republic of China \\ ${ }^{*}$ Corresponding author: Jiaan Der Wang, Department of Pediatrics, Taichung Veterans General Hospital, Taichung, Taiwan, Republic of China. Tel: +886-423592525, Fax: \\ +886-423741359, E-mail: wangjiaander@gmail.com
}

Received: December 3, 2014; Revised: April 28, 2015; Accepted: May 12, 2015

Keywords: Neonate; Stroke; MRI Scan; Wallerian Degeneration

\section{Dear Editor,}

The clinical signs and symptoms of perinatal arterial ischemic stroke (PAIS) may be subtle and nonspecific. Imaging studies provide a good choice for early diagnosis. We report a case of extensive arterial ischemic infarct in a full-term neonate, with a focus on ultrasound and MRI findings.

A 1-day-old male neonate was born at 37 weeks' gestation via cesarean section due to breech presentation. He weighed $2225 \mathrm{~g}$ (10-25th percentile), was $48 \mathrm{~cm}$ in length (50-75th percentile), and had a head circumference of $33 \mathrm{~cm}$ (50th percentile). Apgar scores were 6 and 8 at 1 and 5 minutes, respectively. Maternal hypertension developed 2 weeks before delivery and was treated. After birth, delayed initial crying and cyanosis were noted. Resuscitation was done and the infant was hospitalized for observation. On physical examination, weak sucking and breath holding while crying were noted. Cranial ultrasound on day 3 revealed very mild increased echogenicity over the left thalamus, basal ganglia and periventricular area with ventricular compression (Figure 1). Decreased right upper and lower limb movements were observed on day 4 of life. Repeated cranial ultrasound on day 5 of life revealed more echogenicity over left thalamus, basal ganglia and periventricular area (Figure 1). For further evaluation, magnetic resonance imaging (MRI) and magnetic resonance angiography (MRA) with diffusion-weighted imaging (DWI) were performed on day 5 of life. The T2-weighted brain MRI (T2WI) showed high signal intensity (SI) over the left fronto-parieto-occipital cortical and white matter areas. MRA showed flow reduction over the left MCA branch (Figure 1). Middle cerebral artery (MCA) infarction was diagnosed. Pre-Wallerian degeneration was also noted on DWI, with high SI over the left posterior limb of internal capsule (PLIC), cerebral peduncle and basis pontis (Figure 2).

On the sixth day after birth, progressive ischemic change of finger/toe tips over the four limbs was noted. Heparin therapy was given for 7 days and the skin gradually turned pink. Prothrombin time, activated partial thromboplastin time and survey for coagulation/thrombophilia, including antithrombin-III, protein C and protein S activities, and hyperhomocysteinemia, were unremarkable. For this index case, pregnancy-induced hypertension may be the only risk factor for the stroke.

The patient developed spastic hemiplegia in the following months. MRI performed when he was 8 months of age revealed multicystic encephalomalacia and Wallerian degeneration (Figures 1,2).

The most common feature of PAIS is seizures, accounting for 70-90\%. Additional signs are apnea/cyanosis, lethargy, feeding difficulties, hypotonia and tone asymmetry. However, some infants remain asymptomatic (1). Cranial ultrasound (CUS) is the primary modality of examination when a neonate is suspected to have neonatal encephalopathy. However, it is operator dependent. In one study, the sensitivity was $68 \%$ in the first 3 days and $87 \%$ from day 4 to day 14 (2). In this index case, the echogenicity was not apparent until day 5 of life (Figure 1), so repeated sonographic follow-up was required.

Copyright (C) 2015, Growth \& Development Research Center. This is an open-access article distributed under the terms of the Creative Commons Attribution-NonCommercial 4.0 International License (http://creativecommons.org/licenses/by-nc/4.0/) which permits copy and redistribute the material just in noncommercial usages, provided the original work is properly cited. 

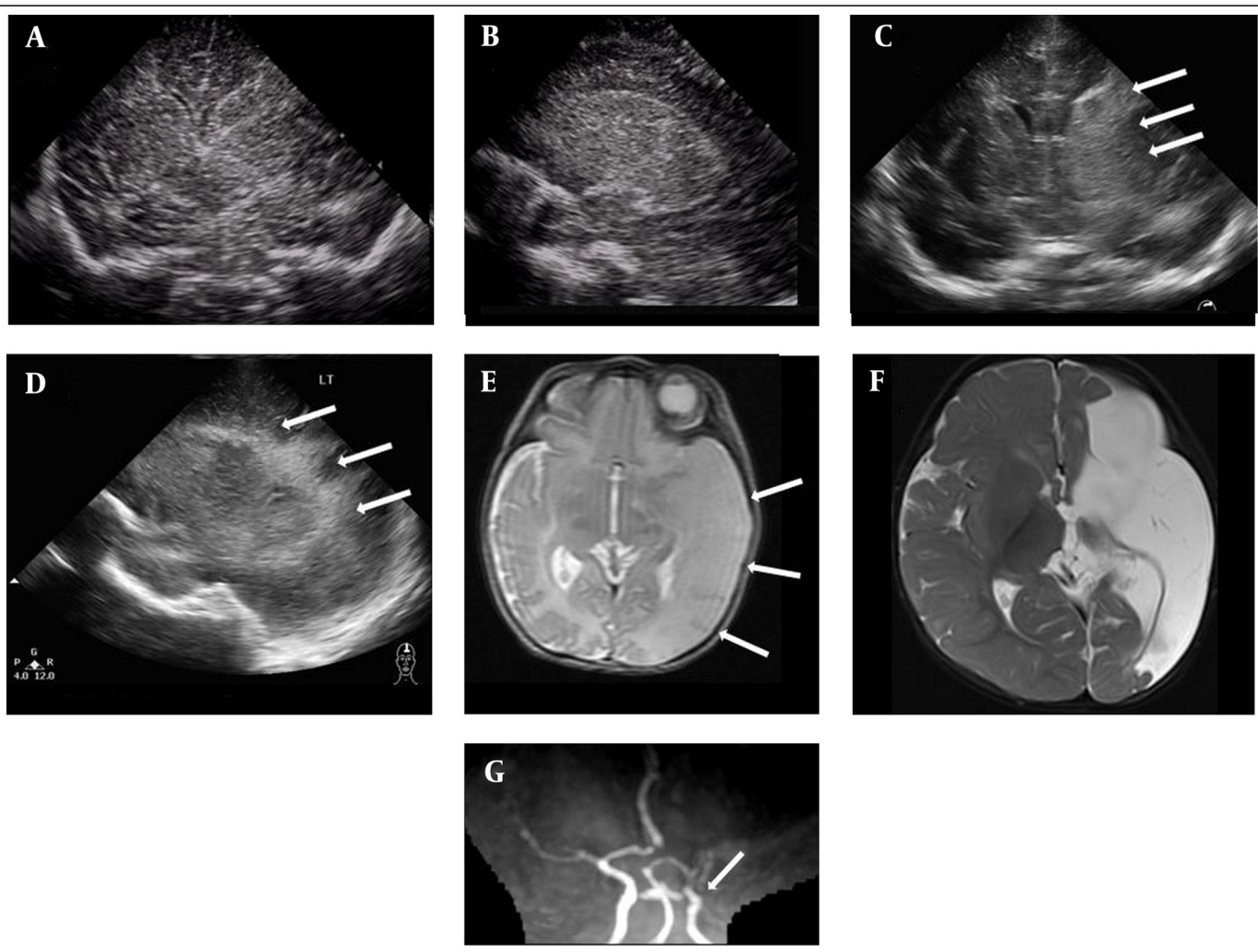

Figure 1. ultrasound scan on day 3 (A, B) and day 5(C, D) of life with coronal and left parasagittal views showed increased echogenicity over the thalamus and basal ganglia. The echogenic area was more apparent on day 5 than day 3 of life (arrow). On day 5 of life, T2WI showed left MCA territory infarction with a "missing cortex sign" (E) (arrow), and MRA showed flow reduction in the left MCA (G) (arrow). Multicystic encephalomalacia was demonstrated on T2WI when the patient was 8 months old (F).
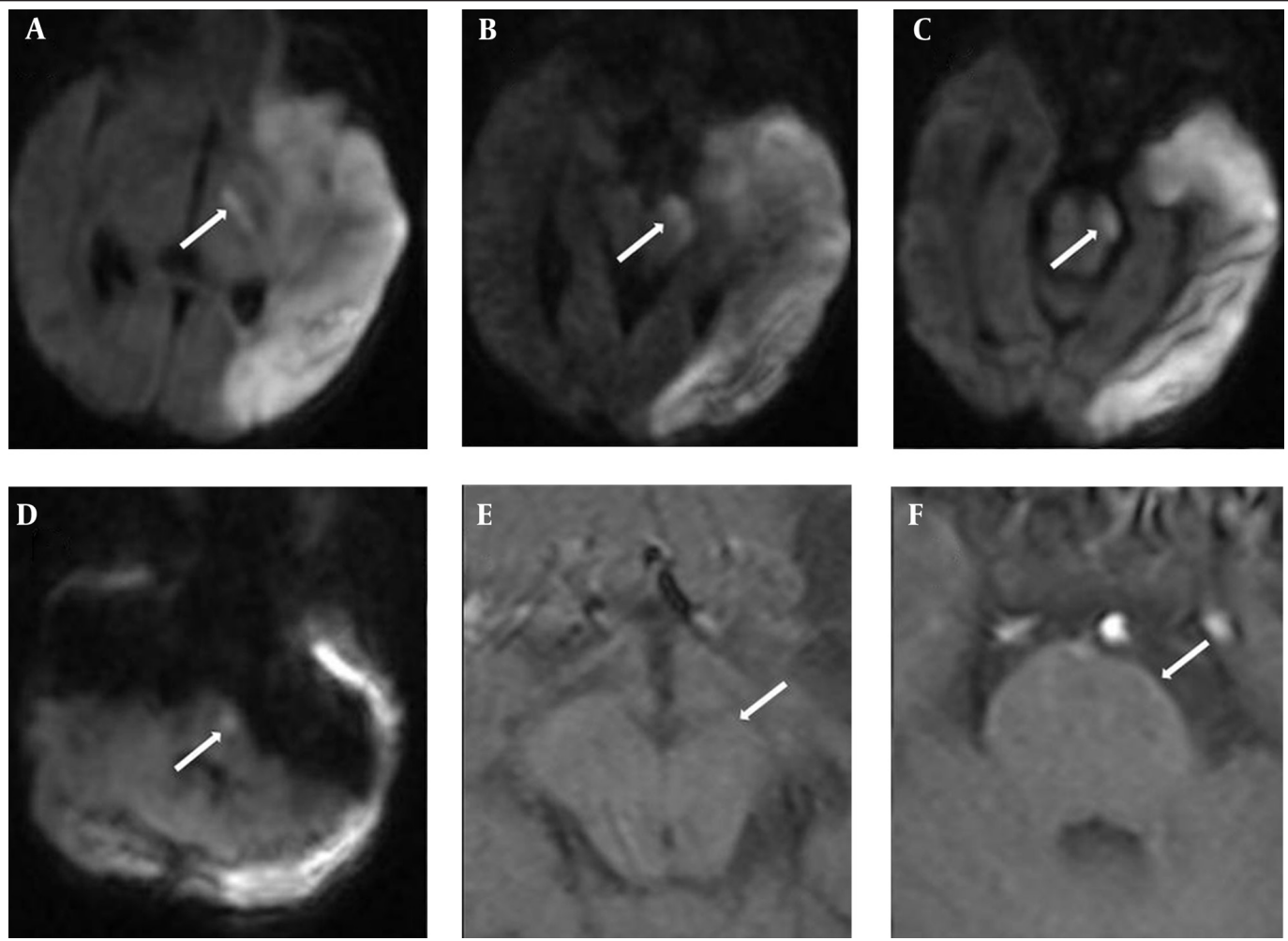

Figure 2. DWI findings on day 5 of Life with DSCT Pre-wallerian degeneration over the PLIC (A), Peduncle (B, C), and basis pontis (D). TIWI performed when the infant was 8 months of age showed volume loss over the left cerebral peduncle (E) and basis pontis (F), compatible with Wallerian degeneration (arrow). 
MRI is the gold standard for diagnosing PAIS. In this index case, the infracted area on T2WI showed reduced contrast with the white matter, which may be due to increasing brightness of the infracted area, leading to absence of cortex (the so-called "missing cortex sign"). MRA of the head provides visualization of great arteries but not small vessels. Carotid dissection is rarely detected in neonates and is associated with birth trauma (3), so a neck MRA may sometimes be needed. In our case, MRA showed asymmetry with reduction in flow through the left MCA and its branches (Figure 1), which was compatible with MCA infarction.

Early Wallerian degeneration (or Pre-Wallerian degeneration), first described in infants by Mazumdar et al. is an acute corticospinal tract injury preceding Wallerian degeneration (4). Early Wallerian degeneration can be detected within the early days of acute AIS, manifesting as increased SI in the ipsilateral descending corticospinal tract (PLIC, cerebral peduncle, basis pontis, and medullary pyramid) on DWI (5). It has been shown that a neonatal descending corticospinal tract (DSCT) DWI signal abnormalities, including the percentage of cerebral peduncle affected, the length of DCST affected, and the relative volume of the DWI DCST lesion, are predictive of motor outcome (5). Similar findings were found on childhood and adult stroke $(6,7)$. In this index case, early Wal- lerian degeneration with subsequent Wallerian degeneration were noted (Figure 2), which correlated with the poor motor outcome.

\section{References}

1. van der Aa NE, Benders MJ, Groenendaal F, de Vries LS. Neonatal stroke: a review of the current evidence on epidemiology, pathogenesis, diagnostics and therapeutic options. Acta Paediatr. 2014;103(4):356-64.

2. Cowan F, Mercuri E, Groenendaal F, Bassi L, Ricci D, Rutherford $\mathrm{M}$, et al. Does cranial ultrasound imaging identify arterial cerebral infarction in term neonates? Arch Dis Child Fetal Neonatal Ed. 2005;90(3):F252-6.

3. Hamida N, Hakim A, Fourati H, Ben Thabet A, Walha L, Bouraoui A, et al. [Neonatal cervical artery dissection secondary to birth trauma]. Arch Pediatr. 2014;21(2):201-5.

4. Mazumdar A, Mukherjee P, Miller JH, Malde H, McKinstry RC. Diffusion-weighted imaging of acute corticospinal tract injury preceding Wallerian degeneration in the maturing human brain. AJNR Am J Neuroradiol. 2003;24(6):1057-66.

5. Kirton A, Shroff M, Visvanathan T, deVeber G. Quantified corticospinal tract diffusion restriction predicts neonatal stroke outcome. Stroke. 2007;38(3):974-80.

6. Domi T, deVeber G, Shroff M, Kouzmitcheva E, MacGregor DL, Kirton A. Corticospinal tract pre-wallerian degeneration: a novel outcome predictor for pediatric stroke on acute MRI. Stroke. 2009;40(3):780-7.

7. DeVetten G, Coutts SB, Hill MD, Goyal M, Eesa M, O'Brien B, et al. Acute corticospinal tract Wallerian degeneration is associated with stroke outcome. Stroke. 2010;41(4):751-6. 